\title{
BROOKHTYEN
}

NATIONAL LABORATORY

BNL-114380-2017-JA

\section{Directionally Interacting Spheres and Rods Form Ordered Phases}

\author{
O. Gang
}

Submitted to ACS Nano

May 2017

Center for Functional Nanomaterials

Brookhaven National Laboratory

\author{
U.S. Department of Energy \\ USDOE Office of Science (SC), \\ Basic Energy Sciences (SC-22)
} manuscript, or allow others to do so, for United States Government purposes. 


\section{DISCLAIMER}

This report was prepared as an account of work sponsored by an agency of the United States Government. Neither the United States Government nor any agency thereof, nor any of their employees, nor any of their contractors, subcontractors, or their employees, makes any warranty, express or implied, or assumes any legal liability or responsibility for the accuracy, completeness, or any third party's use or the results of such use of any information, apparatus, product, or process disclosed, or represents that its use would not infringe privately owned rights. Reference herein to any specific commercial product, process, or service by trade name, trademark, manufacturer, or otherwise, does not necessarily constitute or imply its endorsement, recommendation, or favoring by the United States Government or any agency thereof or its contractors or subcontractors. The views and opinions of authors expressed herein do not necessarily state or reflect those of the United States Government or any agency thereof. 


\title{
Directionally Interacting Spheres and Rods Form Ordered Phases
}

\author{
Wenyan Liu, ${ }^{1, \&}$ Nathan A. Mahynski, ${ }^{2, \&, \#}$ Oleg Gang, ${ }^{1,3,4^{*}}$ Athanassios Z. \\ Panagiotopoulos, ${ }^{2 *}$ Sanat K. Kumar ${ }^{3}$ \\ ${ }^{\&}$ Authors contributed equally to this work \\ ${ }^{1}$ Center for Functional Nanomaterials, Brookhaven National Laboratories, Upton, NY \\ ${ }^{2}$ Department of Chemical and Biological Engineering, Princeton University, Princeton, NJ \\ ${ }^{3}$ Department of Chemical Engineering, Columbia University, New York, NY \\ ${ }^{4}$ Department of Applied Physics and Applied Mathematics, Columbia University, New York, \\ NY \\ \#Current address: Chemical Sciences Division, National Institute of Standards and Technology, \\ Gaithersburg, MD \\ *oleg.gang@columbia.edu, azp@princeton.edu
}

\begin{abstract}
$\underline{\text { Abstract }}$
The structures formed by mixtures of dissimilarly shaped nanoscale objects can significantly enhance our ability to produce nanoscale architectures. However, understanding their formation is a complex problem due to the interplay of geometric effects (entropy) and energetic interactions at the nanoscale. Spheres and rods are perhaps the most basic geometrical shapes, and serve as convenient models of such dissimilar objects. The ordered phases formed by each of these individual shapes have already been explored, however, when mixed, spheres and rods have demonstrated only limited structural organization to date. Here, we show using experiments and theory that the introduction of directional attractions between rod ends and isotropically interacting spherical nanoparticles (NPs) through DNA base pairing, leads to the formation of ordered three dimensional (3D) lattices. The spheres and rods arrange themselves in a complex alternating manner, where the spheres can form either a face-centered cubic (FCC) or hexagonal close-packed (HCP) lattice, or a disordered phase, as observed by in-situ x-ray
\end{abstract}


scattering. Increasing NP diameter at fixed rod length yields an initial transition from a disordered phase to the HCP crystal, energetically stabilized by rod-rod attraction across alternating crystal layers, as revealed by theory. In the limit of large NPs, the FCC structure is instead stabilized over the HCP by rod entropy. We, therefore, propose that directionally specific attractions in mixtures of anisotropic and isotropic objects offer insight into unexplored self-assembly behavior of non-complementary shaped particles.

Keywords: Anisotropic colloids, self assembly, DNA nanotechnology, colloidal crystals, polymorphism, nanoparticles

Understanding and manipulating the self-assembly of nanoscale objects is important for creating materials that exploit the collective properties of the resulting superstructures, with applications in electronics, optics, and catalysis; ${ }^{1-3}$ such properties can be dramatically different from those of the building blocks. ${ }^{4-6}$ Over the past decades, many classes of nanoparticle (NP) superlattices have been assembled using various strategies, e.g., biomolecular recognition, electrostatic forces and entropic effects. ${ }^{7-16}$ Recent studies have shown that incorporation of complex shapes or anisotropic interactions can tremendously enrich the phase behavior of the resulting structures. ${ }^{17-}$ ${ }^{21}$ However, practical limitations in particle engineering often hinder the translation of those ideas into experimental realization. Another strategy to broaden the design space relies on mixing different particles together. Most work to date has used mixtures of differently sized spherical NPs, ${ }^{22-36}$ where the resulting structures are determined primarily by maximum packing considerations. Even greater organizational complexity might be achieved for mixtures of differently shaped objects. ${ }^{37}$ However, at present, such increased structural diversity is also accompanied by phase separation and the formation of disordered states, as has been shown for mixtures of perhaps the simplest shapes: rods and spheres. ${ }^{38-40}$ Experiments have revealed that rods and spherical particles can assemble into layered structures by maximizing their packing density even in the absence of interparticle interactions. While these previous findings were the result of either entropic or non-directional attraction effects, here we reconsider sphere-rod systems by including basic directional attractions, commensurate with a rod geometry, provided by the rod ends. 
We show here that such a simple modification stabilizes phases not previously observed for mixtures of rods and spheres: close-packed organizations of NPs, either hexagonal close-packed (HCP) or face-centered cubic (FCC) lattices, both accompanied by a complex organization of rods. These findings demonstrate a way to engineer binary lattices of shaped blocks via selectively chosen anisotropic attractive interactions. Furthermore, the mechanism shown here for the formation of the FCC and HCP lattices might shed light on the long-debated question about the appearance of these phases. ${ }^{41-42}$ While colloidal systems are often considered the simplest, yet insightful, models of their atomic counterparts, our study demonstrates that the addition of anisotropic interactions might be crucial for mimicking the behavior of atomic organizations more closely. Our experimental system was constructed from spherical NPs coated with single stranded DNA (ssDNA) and rigid DNA bundles capped with ssDNA, the latter serving as rods with customizable end interactions. Small angle x-ray scattering (SAXS) was used as the in-situ method for probing the structural behavior of the assembled systems. In parallel, computational studies, which employed molecular dynamics (MD) simulations with realistic parameters, complemented by a simple mean-field theory, reveal the mechanism of crystal formation and the range of each polymorph's stability.

\section{$\underline{\text { Results and Discussion }}$}

DNA-based approaches were recently demonstrated as a powerful platform to assemble nanoparticle clusters and lattices. ${ }^{7,43-46}$ Besides the modularity of these assembly methods, they also permit the exploration of fundamental physical phenomena in the organization of matter, such as the interplay between entropic and enthalpic effects, and the role of directional forces and other relevant physical factors. ${ }^{47}$ For example, the profound effect of the DNA "core-shell" design has been demonstrated for DNA-coated spherical nanoparticles, and both the entropic and enthalpic contributions to the formation of ordered phases have been revealed. ${ }^{7,48-49}$ We note that when shells are composed of a large number of DNA chains, the interparticle interactions are isotropic. In such a case, the binary phase diagram is mainly determined by particle size ratios and stoichiometry, ${ }^{11,49-51}$ although collective polymeric effects can lead to peculiar assembly pathways with an anisotropic character. ${ }^{52}$ However, in the case we present here, we show how a small number of rods hybridized on a particle can imbue the shell with a discrete, anisotropic 
character. Such shells can provide directional interparticle interactions, and may affect the phase diagram drastically. This method of inducing anisotropic bindings at the nanoscale stands in contrast to more conventional routes, which are typically realized via particle shape. For example, it was recently demonstrated that nanoscale cubes and octahedra coated with DNA could enforce organizations of spherical particles via face-directed interactions ${ }^{53}$ into lattices whose symmetry is dominated by the geometry of the anisotropically shaped blocks. Alternatively, shaped DNA constructs $^{54}$ can link particles through vertex-NP bindings in topologically diverse ways resulting in a variety of lattices ${ }^{55-56}$ with structures determined by the geometry of the DNA constructs, rather than the particle shapes. Molecular level effects, such as collective chain interactions, were shown to induce a linear arrangement of alternating rods and spheres. ${ }^{52}$ The diversity in structural organizations of spheres linked via different motifs is far from being completely understood. In this work, we address this challenge by focusing on a rod-sphere system with simple directional interactions provided by the rods. We show that phase separation, common for such binary mixtures, can be overcome in favor of self-assembly. Moreover, we illustrate assembly into complex lattices, in which the rods are organically incorporated into phases native for isotropic spherical objects. Our study reveals that the formation of these specific lattices is determined by the interplay of entropic and anisotropic enthalpic effects, which is controlled by the ratio of the particle diameter and rod length, stoichiometry, and energetics of rod-rod end group interactions.

Figure 1 illustrates the experimental system comprised of spherical gold NPs coated with ssDNA which is terminated with recognition motifs, and the DNA rods, each formed by a bundle of six double helices (6HB, see Figure S1 for detailed design) that contains, at its termini, 6 ssDNA strands complementary to the ssDNA on the NPs. We grafted gold NPs (core diameters, $t$, are 6.0 $\mathrm{nm}$ and $8.7 \mathrm{~nm}$ ) through the thiol-terminus of ssDNA. On the NP surface these ssDNA strands consist of a 6-nucleotide (nt) recognition part that can hybridize with its complementary sequence on the $6 \mathrm{HB}$, and a flexible spacer $\left(S_{n}\right)$ of different lengths $(\mathrm{n}=6,14,24,29,44-\mathrm{nt})$, which functions to separate the recognition part from the NP surface, and to adjust the effective NP diameter; these systems are referred to $P_{t} S_{n}$, where $t$ is the particle core diameter in nanometers and $n$ is the number of nucleotides in the spacer. Thus, the NPs, evenly grafted with DNA, can be considered to be isotropically interacting objects, while the attachment of the rods 
to the NPs can occur only at the rods' termini. We assembled the $21 \mathrm{~nm}$ long 6HB DNA structure from 20 DNA strands (see Methods) through a thermal annealing process, and subsequently verified it by native polyacrylamide gel electrophoresis (PAGE, see Figure S2). Such a design yields objects with extremely high persistence lengths, approximately $1 \mu \mathrm{m},{ }^{57-61}$ and therefore, a $6 \mathrm{HB}$ structure can be assumed to be a rigid rod in the context of our studies. The assembly of the experimental systems was conducted in a buffer environment containing 12.5 $\mathrm{mM}$ of $\mathrm{Mg}^{2+}$, which is required for the stabilization of the $6 \mathrm{HB}$ structure. We stress that the interaction between rods and NPs is highly specific since it is encoded by the complementarity of the corresponding DNA sequences. In addition to the strong NP-rod base pairing interactions, a weak non-specific interaction also exists between the ends of two rods due to the divalent ion $\left(\mathrm{Mg}^{2+}\right)$ mediated attractions of polyelectrolytic ssDNA chains ${ }^{62-66}$ (see Supplementary Figure S3). Experimentally, NP-rod assemblies were investigated by in-situ synchrotron-based $\mathrm{SAXS}^{7}$ (beamline X9, NSLS, BNL, see Methods) to probe the effects of NP size and the stoichiometric ratio between the $6 \mathrm{HB}$ rods and the DNA-coated NPs. The systems were assembled through a thermal annealing process to achieve an equilibrium state prior to the scattering studies (see
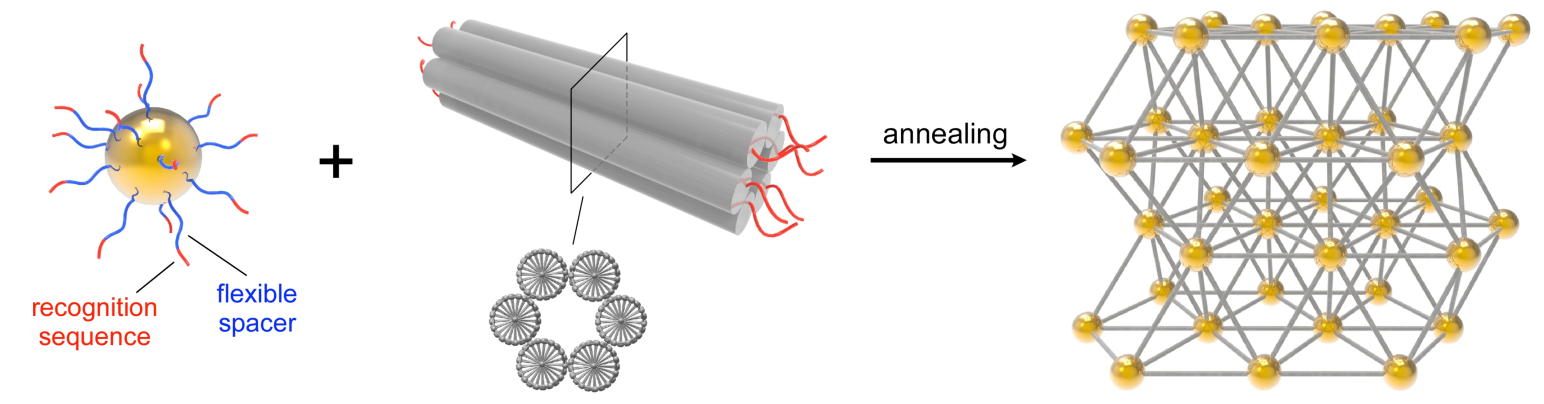

Figure 1. Schematic drawing of the rod-sphere system: rods, formed from a six-helix DNA bundle (6HB), mediate interactions of spherical DNA coated gold nanoparticles. Gold nanoparticles (AuNPs) were functionalized with thiol-ssDNA composed of a flexible spacer (blue) and a recognition sequence (red) that can hybridize with its complementary counterpart (red) on the 6HB rod. DNA-capped AuNPs can be assembled into different structural arrangements by adjusting the length of the flexible spacer and the $6 \mathrm{HB} / \mathrm{AuNP}$ size ratio.

Methods).

In our molecular dynamics simulations, we considered a dilute mixture of rods and spheres with volume fractions below 0.025 . To mimic experimental conditions discussed above, we assigned 
attractive interactions between rod ends and sphere surfaces, and between different rod ends. Inspired by experiments, we assumed the former interaction to be twice as favorable as the latter, such that the non-specific rod-rod interaction was weaker than the DNA-encoded rod-sphere interaction. Theoretical arguments reveal that the ratio between these two interactions does not qualitatively affect our results, it merely changes the quantitative temperature ranges over which each structure is formed. Directionality in rod-rod and rod-NP interactions was imposed by surrounding the ends of the rods with a repulsive "shell" which prevented the ends from energetically interacting simultaneously with multiple other ends, and with NP surfaces at oblique angles ( $c f$. Methods). We systematically varied the diameter of the spheres, while holding the rod length fixed $\left(l_{\text {rod }}=6 \sigma\right.$, where $\sigma$ is the rod diameter which defines the smallest characteristic length scale in the system).

We first studied the smaller NP (6.0 nm core diameter) with a flexible ssDNA spacer $(\mathrm{n}=29)$ as a

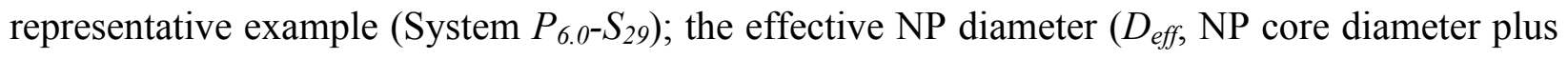
the spacer shell) in this case is estimated ${ }^{9}$ to be about $22.6 \mathrm{~nm}$ (see Supplementary Material). The mixtures were prepared at different nominal rod:NP stoichiometric ratios of 1:10, 1:5, 1:2, 1:1, 2:1, 5:1, and 10:1 and were annealed ( $c f$. Methods). The systems formed macroscale aggregates (0.1-0.3 $\mathrm{mm})$, visible to the naked eye, and precipitated. We conducted in-situ SAXS experiments, and the $2 \mathrm{D}$ scattering pattern was converted into a $1 \mathrm{D}$ structure factor, $\mathrm{S}(q)$, by azimuthal integration and dividing by the NP form factor. We then normalized the peak positions based on the $q$ value of the first ring (where $q$ is the wave vector). Our SAXS measurements revealed only disordered aggregates for ratios below 5:1, as indicated by the broad and illdefined structure factors (Figure 2a). This aggregation behavior at low ratios indicates that both ends of the $6 \mathrm{HB}$ rods connect to the NPs with high efficiency, ${ }^{9}$ thus, particles are able to link as many rods as necessary to achieve an aggregated, although disordered, state. Obviously, this can occur only if the ratio within the aggregate differs significantly from the nominal one. Indeed, we observed that the solution remained pink-colored during aggregation at low ratios, which confirmed that only a fraction of particles participated in the aggregation. In contrast, at higher ratios the solution became colorless, which is indicative of all NPs being consumed in the NProd hybridization reaction. In conjunction with this, when the ratio was increased to 5:1 and higher, a well-ordered structure emerged, as indicated by an increased number of diffraction 
peaks and their narrow widths. $S(q)$ remained practically unchanged even when the ratio was increased to 10:1, indicating the structural stability over a broad range of stoichiometric ratios.

Figure $2 \mathrm{~b}$ depicts a typical 2D SAXS pattern (inset) and the corresponding $S(q)$ for $P_{6.0}-S_{29}$ at a stoichiometric ratio of 6:1. $S(q)$ reveals a remarkable degree of long-range order, as manifested by more than 20 orders of resolution-limited Bragg's peaks. Analysis of the peak positions yields a ratio $q_{\mathrm{n}} / q_{1} \approx 1: \sqrt{9 / 8}: \sqrt{41 / 32}: \sqrt{17 / 8}: \sqrt{3}: \sqrt{113 / 32} \ldots\left(q_{1}=0.0192 \AA^{-1}\right)$, which is in excellent agreement with the HCP lattice (inset; blue curve in Figure $2 b$ is the scattering from the HCP structure). We also note that the relative peak intensities also are in good agreement with expectations. The experimental center-to-center nearest-neighbor interparticle distance $d$ $\left(d=4 \pi / \sqrt{3} q_{1}\right) \approx 38 \mathrm{~nm}$ was obtained based on the first peak position. This value is smaller than the sum of the NP effective diameter and rod length, thus suggesting that the hybridization of rods between NPs occurs off-axis, which is highly possible due to the low density of rods on the NP surface. In this case rod orientation and its displacement towards a particle periphery contribute to the established interparticle distance. We note that the observed value is close to the estimations (see supporting material) based on the systems with double stranded linking motifs, ${ }^{67,68}$ indicating that molecular alignment is an important factor determining NP separations. 

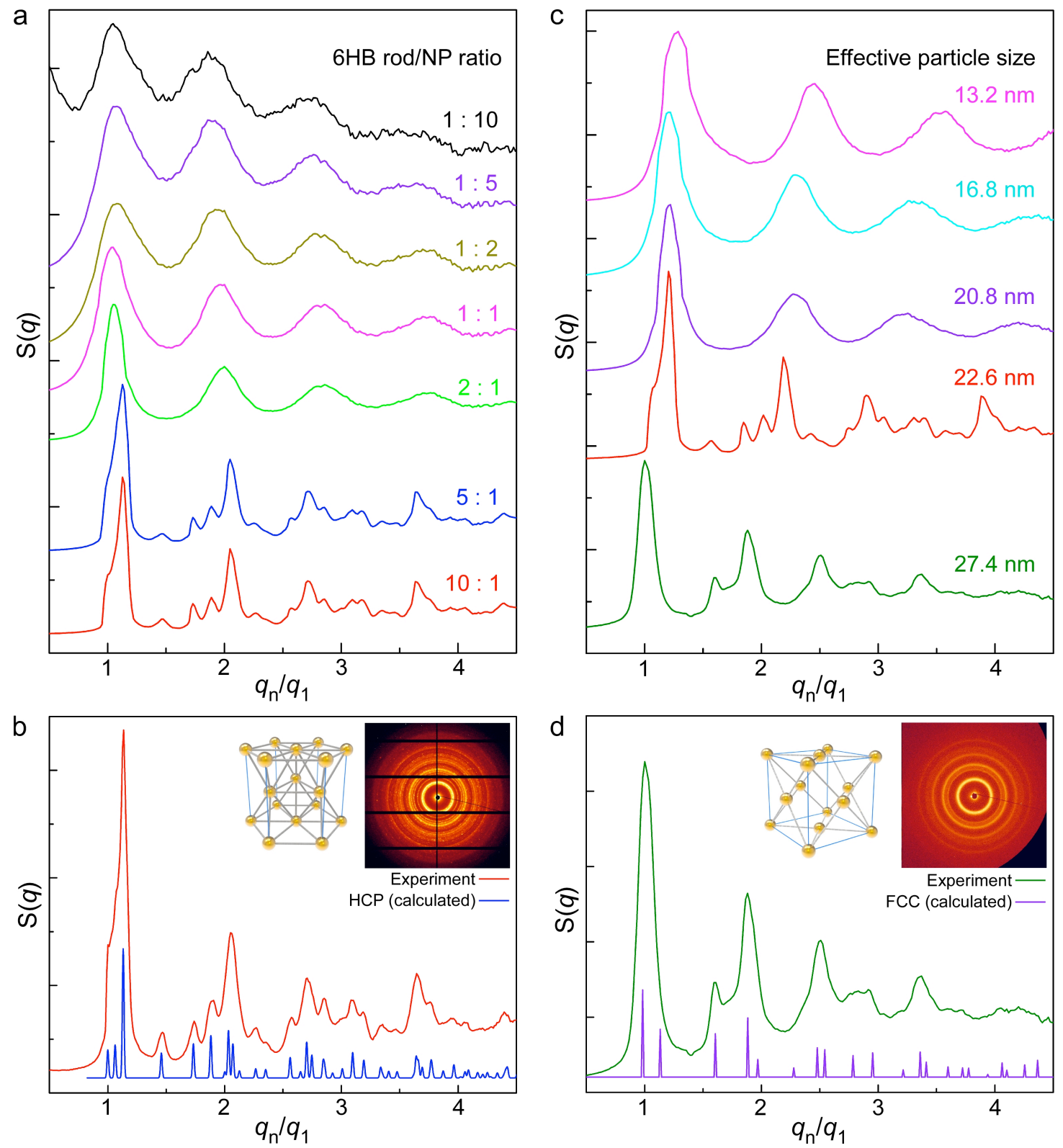

Figure 2. SAXS patterns from the analyzed systems. a, Ratio-dependence of SAXS patterns from the system $P_{6.0}-S_{29}$. b, 2D (inset) and integrated 1D patterns for the system $P_{6.0}-S_{29}$ at a 6HB:NP ratio of 6:1. The result shows a HCP crystal structure. The calculated scattering profile for HCP is shown in blue. c, SAXS patterns of annealed systems with different effective particle sizes at a $6 \mathrm{HB}: \mathrm{NP}$ ratio of 10:1. As the effective particle size was increased, the studied systems showed a phase transition from a disordered state to a HCP crystal, then from a HCP to a FCC crystal. d, 2D (inset) and integrated 1D patterns for the system $P_{6.0}-S_{44}$ (effective particle size: $27.4 \mathrm{~nm}$ ) at a 6HB:NP ratio of 10:1. The result shows a FCC crystal structure. The calculated scattering profile for FCC is shown in violet. The calculated SAXS patterns were generated using VESTA (http://jp-minerals.org/vesta/en) and GDIS (http://gdis.sourceforge.net/). 
We further examined the impact of the effective particle size on the crystalline phase diagram by varying either the length of the NP-capping DNA or the particle core size. Figure 2c summarizes the structural changes observed for the systems $P_{6.0} S_{n}$ at a $6 \mathrm{HB}: \mathrm{AuNP}$ stoichiometric ratio of 10:1. It can be seen that when the effective particle diameters are small, the systems $\left(P_{6.0}-S_{6}\right.$, $P_{6.0}-S_{14}$, and $\left.P_{6.0}-S_{24}\right)$ are in a disordered phase, as evidenced by a limited number of broad SAXS peaks. However, when the effective particle size increases to $D_{\text {eff }} \approx 22.6 \mathrm{~nm}\left(P_{6.0}-S_{29}\right)$, the system exhibits a highly ordered HCP crystal phase as confirmed by the excellent agreement between the experimental data and the theoretical predication. With further increase in the particle size to $D_{\text {eff }} \approx 27.4 \mathrm{~nm}$, the rod-sphere system $\left(P_{6.0}-S_{44}\right)$ still shows a well-defined crystalline phase, however, its SAXS diffraction pattern is clearly different from that of the system $P_{6.0}-S_{29}$. Analysis of the diffraction peaks reveals that both the position ratios $\left(q_{n} / q_{1} \approx 1\right.$ : $\left.\sqrt{4 / 3}: \sqrt{8 / 3}: \sqrt{11 / 3}: 2 \ldots\left(q_{1}=0.0177 \AA^{-1}\right)\right)$ and the relative peak intensities index to a facecentered cubic (FCC) structure (Figure 2d). The center-to-center nearest neighbor interparticle distance $d$, as calculated using the $q$ value of the first peak $\left(d=\pi \sqrt{6} / q_{1}\right)$, is approximately $43 \mathrm{~nm}$, which agrees well with the estimated interparticle distance $(\approx 42.5 \mathrm{~nm})$. The systems containing NPs with a core diameter of $8.7 \mathrm{~nm}$ also formed a FCC phase at larger effective particle sizes (see Supplementary Material). We summarize in Figure 3 the experimentally observed phase diagram, where the effective NP size (core and DNA shell) is plotted as a function of the nominal stoichiometric ratio of the $6 \mathrm{HB}$ to the NP. With increasing effective NP size, a transition from disorder to HCP to FCC occurs, however, these transitions only take place if the stoichiometric ratio of linkers to NPs is larger than approximately 5:1. The FCC phase was observed for particles with larger effective diameters, either with $6 \mathrm{~nm}$ or $8.7 \mathrm{~nm}$ gold cores, while the HCP was present only for smaller NPs. These observations clearly indicate that the effective particle size has a strong influence on the phase behavior of NP-rod assemblies, possibly through the particle to rod size ratio. To further elucidate this question we performed a computational investigation. 


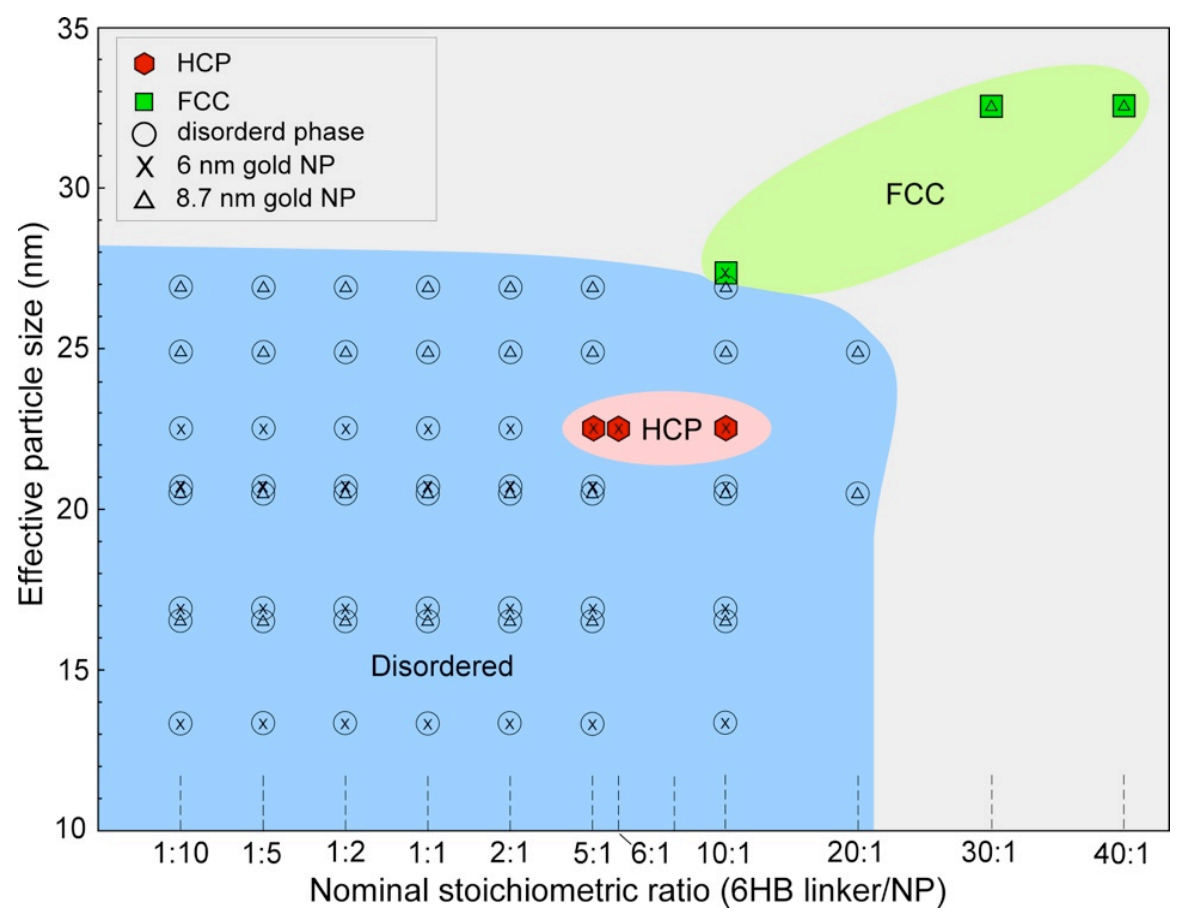

Figure 3. Experimental phase diagram. Summary of the experimentally observed phase behavior of NP assemblies mediated by $6 \mathrm{HB}$ rod linkers for various effective particle sizes (core and DNA shell) at different nominal stoichiometric ratios of linkers to NPs. The sizes of the gold cores are as shown.

The formation of HCP and FCC organizations of spherical particles interacting via directionally attractive rods is outright surprising in light of previous experiments and theory which suggest that these systems should only form lamellae, driven primarily by the dominance of rod packing. Moreover, while the formation of a FCC lattice could perhaps be rationalized based on the general behavior of hard spheres (and also spheres under attractive conditions), the formation of a stable HCP phase is altogether unexpected. We conducted detailed simulations to understand the mechanism leading to the formation of these HCP lattices (Figure 4). In MD simulations of the smallest NPs (core diameter, $\sigma_{c}=4.0 \sigma=16 \mathrm{~nm}$ ) we observed a peak in the radial distribution function, $\mathrm{g}(r)$, at $r=l_{\text {rod }}+\sigma_{c}$ while long-range order was suppressed. Consistent with experiments which yield amorphous aggregates, spheres in this system were found paired into dumbbells, owing to rod end-NP attractions, but no significant higher order structure was found. However, when $\sigma_{c}=6.95 \sigma$ we observed peaks in the radial distribution function consistent with the formation of a hexagonal close-packed (HCP) crystal. Specifically, we point out the characteristic peaks at $r /\left(l_{\text {rod }}+\sigma_{\mathrm{c}}\right)=\sqrt{ }(11 / 3) \approx 1.91$ and $r /\left(l_{\text {rod }}+\sigma_{\mathrm{c}}\right)=2$. While the latter 
peak is always present in a close-packed crystal, the former is only present when alternating (next-nearest neighbor, not nearest neighbor) stacking planes of the crystal repeat (Figure 4), i.e., the HCP structure. For larger spheres, when $\sigma_{\mathrm{c}}=10.0 \sigma$, the peak at $\sqrt{ }(11 / 3)$ was always suppressed and the system instead adopted the FCC geometry where alternating planes are offset by $60^{\circ}$. Icosahedra were also commonly observed in these systems, but have a similar offset between planes.

We only observed HCP crystals in our MD simulations over a narrow range of size ratios that yielded crystals with an interplanar spacing commensurate with the size of a pair of rods. The interplanar distance between sphere surfaces for close-packed rod-mediated colloidal crystals is given by $D=2 \sqrt{\frac{2}{3}}\left(\sigma_{c}+l_{\text {rod }}\right)-\sigma_{c}$ . When $2<D / l_{\text {rod }} \lesssim 2.25$, which occurs when $0.6<\sigma_{c} / l_{\text {rod }} \lesssim 1$, the free ends of two rods attached to the surfaces of NPs in next-nearest neighbor stacking planes which face each other are capable of energetically interacting when these planes eclipse each other, i.e., in the HCP configuration. However, in the FCC stacking pattern, such next-nearest plane energetic interactions are not possible because the planes are staggered. Therefore, the HCP crystal is strongly stabilized over the FCC by directional energetic interactions. This behavior was only observed when the rods' interaction with the colloids was directionally specific; that is, rods and spheres could only energetically interact when they approached each other "head on" as occurs when their complementary strands hybridize, whereas oblique interactions were not allowed. In contrast, only amorphous aggregates formed when the rods were allowed to interact with other species in a spherically symmetric fashion. This was tested by removing the repulsive "shell" around the rod ends shown in Figure 4, which is responsible for imposing such directionality to mimic the nature of the DNA-based interactions. When the interplanar surface separation is less that $2 l_{\text {rod }}$ $\left(\sigma_{c} / l_{\text {rod }}<0.6\right)$, both crystals' interiors are highly frustrated environments for the rods attached to the NP surfaces, due to steric rod-rod overlap, thus making both crystals difficult to form. While this accounts for the amorphous structure observed with small NPs, it does not explain why the FCC crystal is apparently more stable when the interplanar distance is increased, i.e., for larger NPs. To understand this result we turn to a simple model. 
We posit that the primary difference between the different polymorphs is the interference that exists between rods attached to the surfaces of NPs in next-nearest stacking planes. Differences
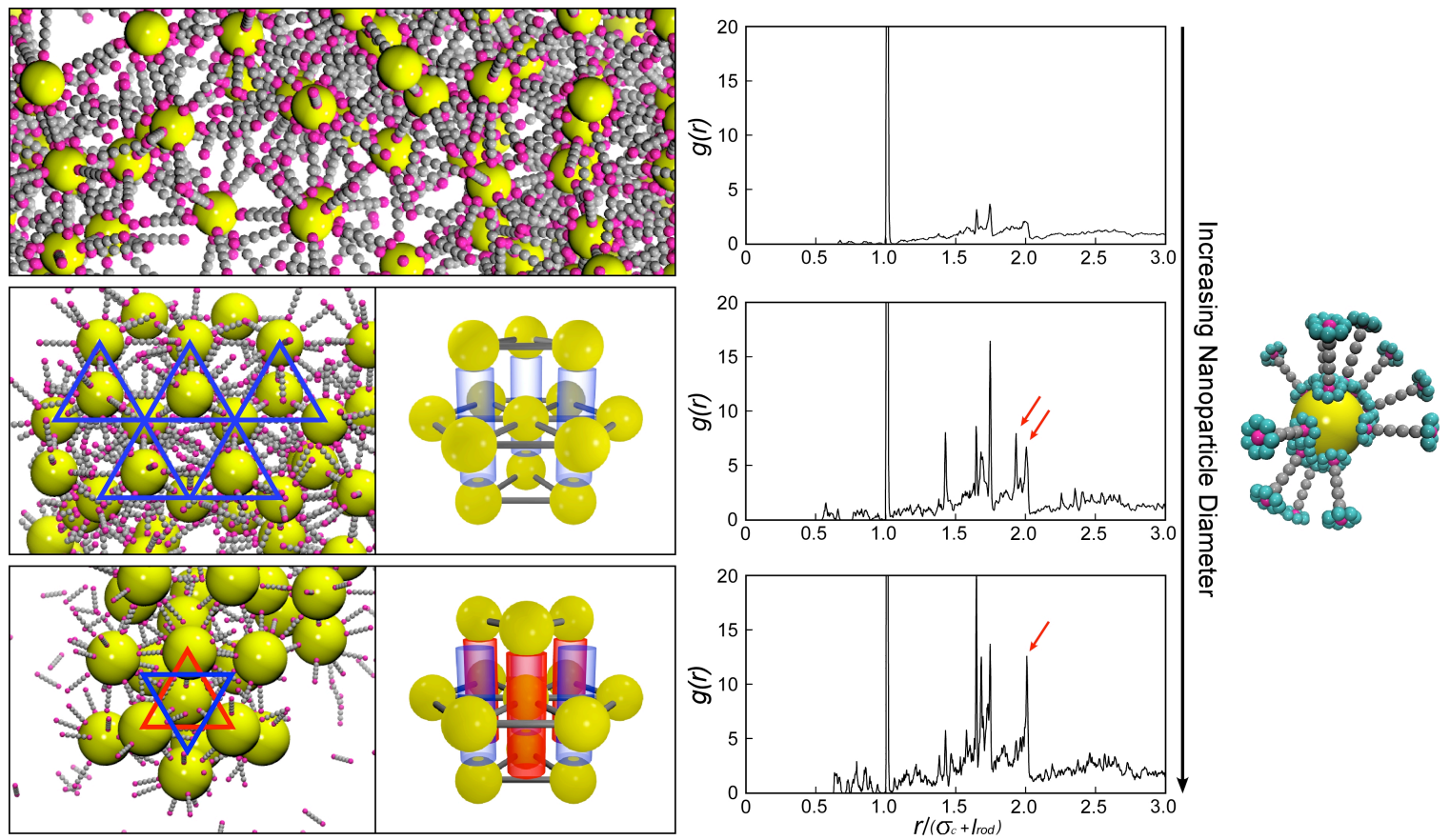

Figure 4: $\mathrm{MD}$ results for the pair correlation function, $\mathrm{g}(r)$, between a rod and NPs at $T^{*}=$ 0.12, and corresponding simulation snapshots with increasing NP diameter. (See Supplementary Material for more detail.) The cartoon at the right depicts a NP (yellow) surrounded by rods whose ends (magenta) interact both with other ends and the NP surface. The repulsive cyan "shell" around the ends imposes directionality by preventing other ends from interacting obliquely and preferentially orients the rod normal to the surface of the colloid. The shells otherwise do not interact with the system and so are neglected for visual clarity in the rest of this figure. (Top Row) For small NPs $\left(\sigma_{\mathrm{c}}=4 \sigma\right)$, only amorphous aggregates were observed; the $\mathrm{g}(r)$ illustrates no long-range ordering and only minimal shortrange order below $r /\left(\sigma_{\mathrm{c}}+l_{\text {rod }}\right)=2$. (Middle Row) For intermediately sized colloids $\left(\sigma_{\mathrm{c}}=\right.$ $6.95 \sigma$ ) the system begins to display HCP ordering where next-nearest stacking layers eclipse each other, as evidenced by the emerging second peak in $\mathrm{g}(r)$ at $r /\left(\sigma_{\mathrm{c}}+l_{\text {rod }}\right)=\sqrt{ }(11 / 3) \approx$ 1.91. The interplanar spacing in which rods adsorbed on the NP surfaces can fluctuate is graphically depicted by the blue cylindrical "channels" in the cartoon. This peak emerges as a consequence of the NPs at opposite ends of neighboring channels, which only appear as such in the HCP configuration. (Bottom Row) For large colloids $\left(\sigma_{\mathrm{c}}=10 \sigma\right)$ this peak is absent and the HCP character is entirely suppressed. Instead, the system exclusively adopts configurations where these planes are staggered (both red and blue channels between the layers) resulting in FCC structures or icosahedral intermediates.

at this level are expected to be more significant than nearest-neighbor interactions since the 
coordination number in close-packed lattices is identical, with only a small difference in relative orientation of the NPs in the nearest-neighbor shell. In the FCC crystal, since alternating planes are staggered (ABC pattern), rods adhered to the surface of NPs in these planes can only interact obliquely, whereas in the HCP, because the planes are eclipsed (ABAB pattern), a pair of rods can interact with each other directly across alternating layers, i.e., through the blue channels indicated in Figure 4. Consequently, we focus exclusively on the behavior of these rods in channels, which provide the different polymorphs. If we reduce this to a one-dimensional model, considering a lattice onto which a pair of rods is placed, we can contrast the two crystals (see Methods). Using statistical mechanics, we calculated the free energy of this one-dimensional "toy" model by accounting for rod end-rod end attractions, which are possible only in the HCP crystal, and the fact that the FCC provides essentially twice the volume for the rods to exist in (both red and blue channels in Figure 4 for the FCC, whereas the HCP has only blue ones). Representative results (Figure 5) show precisely the same transitions seen in the simulations, i.e., from an amorphous state to a HCP crystal, then from HCP to FCC as the sphere diameter is increased ( $c f$. Figure 3). For very small colloid diameters the distance between the sphere faces across alternating layers is smaller than twice the rod length. The HCP crystal is thus strongly disfavored due to rod-rod overlap. However, in this regime the FCC crystal is also a highly crowded environment for the rods, suggesting that an amorphous state is more favorable than either crystal. For larger colloids, when $D \approx 2 l_{\text {rod, }}$, the entropy of a pair of rods in the HCP state is small, having essentially only one possible configuration. Despite this low entropy state, the short-ranged attractive interactions between the ends of the rods, which extend across to the alternating layers, energetically stabilize the HCP crystal. However, rod entropy quickly destabilizes this state in favor of the FCC for larger NPs. Because this crystal offers the rods twice as many channels, the translational entropy of these rods is greatly increased over the case where they share the same channel (HCP). Without a significant energetic incentive, the FCC provides a lower free energy configuration for the rods than its HCP counterpart. This model explains why the FCC crystal is definitively favored at larger colloid diameters $\left(D>>2 l_{\text {rod }}\right)$. Previous work on hard sphere colloidal crystals has revealed a marginal entropic favorability of the FCC over the HCP by $\sim 0.001 k_{\mathrm{B}} T$ per sphere. However, this difference does not strongly favor one polymorph over the other, and consequently, both the HCP and FCC are often formed from crystallizing suspensions of colloids. ${ }^{69-70}$ In contrast, in the framework of this simple model, 
the inclusion of these rods at $T^{*}=0.10$ results in a free energy difference of $\approx 0.15 \varepsilon$ per pair of rods in favor of the FCC for large colloids, while near $D \approx 2 l_{\text {rod }}$ the HCP is favored by $\approx 0.30 \varepsilon$ per pair of rods. In simulations, we observed that most rods condensed on the surface of the spheres at this temperature, and a stoichiometry of rods to colloids of 10:1 suggests a difference as high as approximately $0.75 \varepsilon$ per sphere in favor of the FCC for large colloids due to rod entropy, and approximately $1.5 \varepsilon$ in favor of the HCP at intermediate diameters due to rod energy. Of course, some rods are used to bind the lattices together and so do not participate in producing this free energy difference, however, even a single pair of rods participating in interplanar bonding provides a difference three orders of magnitude in excess of that inherent to closepacked lattices.

\section{Conclusions}

In summary, the inclusion of directional attractions in the canonical example of a simple rodcolloid mixture results in intriguing phase behavior. While both athermal and directionally isotropically interacting mixtures yield only lamellar structures, where rod packing dominates the behavior, these new cases yield disordered, hexagonal closed packed, and face centered cubic crystals. A combination of theory, simulation, and experiment suggests that these different structures represent a subtle balance between the energy and entropy of the rods in the different possible self-assembled structures. We postulate that the introduction of even more complicated shapes should lead to the rational self-assembly of more exotic lattices. 

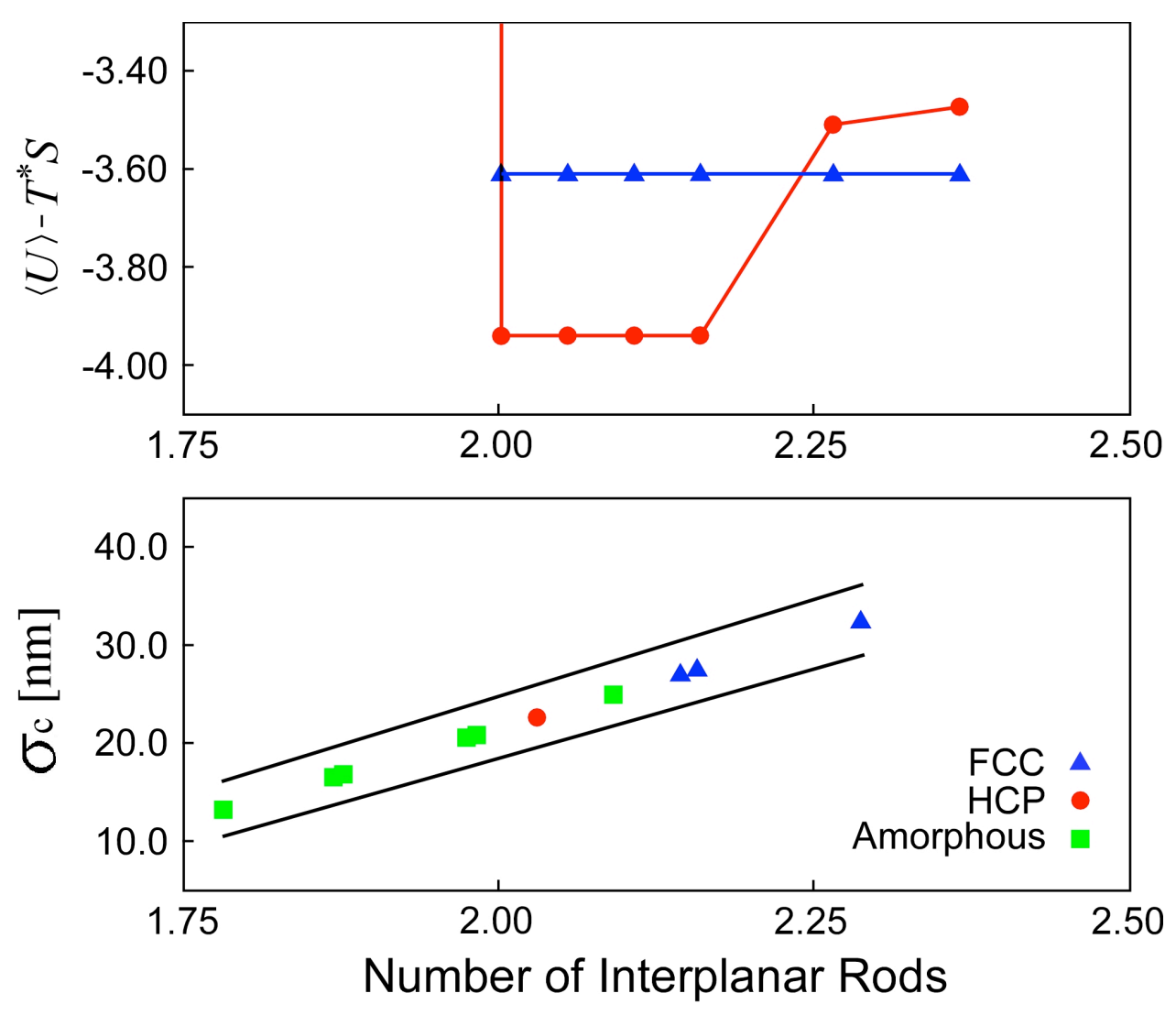

Figure 5: (Top) Free energy of the rod and sphere lattice model at $T^{*}=0.10$ for a fixed rod length. The interplanar spacing increases with NP diameter, $\sigma_{c}$, allowing more rods to exist between the next-nearest stacking layers. The FCC configuration affords the rods twice as many "channels" (both red and blue, $c f$. Figure 4) between the layers and is entropically biased over the HCP polymorph when the number of rods that can be accommodated between these layers is large. However, when approximately two rods fit between the layers, the end-end attractions between rods which are still attached to the surfaces of the NPs (permissible only in the HCP configuration) energetically stabilize this crystal. When these rods overlap because the spacing is less than two rods, the model is no longer predictive for the HCP, but we anticipate both crystals will be disfavored relative to an amorphous state because the rods will experience an increasingly crowded environment in both polymorphs. (Bottom) Experimental results at similar conditions. We observe a transition from amorphous aggregates, to HCP, to FCC crystals, just as the model nearly quantitatively predicts. Because the lattice model does not account for the softness of the NP spheres or fluctuations in the binding of the rod to the NP surface, we estimate an upper and lower bound for the number of interplanar rods by fixing the lattice constant at $\sigma_{\mathrm{c}}+21 \mathrm{~nm}$ (which corresponds to the full hybridization of the ssDNA on the ends of the rods and NP surface) and use an effective rod length which fluctuates between full hydridization of the NP surface $\left(l_{\text {rod }}=21 \mathrm{~nm}+\right.$ $1.98 \mathrm{~nm})$ and none $\left(l_{\text {rod }}=21 \mathrm{~nm}+2 * 1.98 \mathrm{~nm}\right)$. We report the experimental behavior above at the average between these two bounds. 


\section{$\underline{\text { Methods }}$}

\section{Molecular dynamics (MD) simulations}

Model: We model the DNA linkers as rigid rods composed of six tangent spheres, with a radial "shell" (S) around the end beads (E). Each bead has a diameter, $\sigma=1=4.00 \mathrm{~nm}$, which is the length scale we use to non-dimensionalize all units. The length of the rod is therefore $l_{\text {rod }}=6 \sigma$ $=24.00 \mathrm{~nm}$ which closely mimics experiments. These beads are treated as a single rigid body, where the ends thermally interact with each other and with the DNA-coated nanoparticle colloids (C). These interactions are a sum of a Yukawa-like potential, which provides as attractive tail, and the Weeks-Chandler-Andersen (WCA) potential, which provides a short-ranged repulsion ( $c f$. Figures 1 and 2).

$$
\begin{gathered}
U_{\text {tot }}(r)=U_{w c a}(r)+U_{y u k}(r) \\
\infty \quad r \leq \Delta_{i j} \\
\begin{array}{c}
U_{w c a}(r)=4 \varepsilon_{i j, w}\left(\left(\frac{1}{r-\Delta_{i j}}\right)^{12}-\left(\frac{1}{r-\Delta_{i j}}\right)^{6}\right)+\varepsilon_{i j} \quad \Delta_{i j}<r<2^{1 / 6}+\Delta_{i j} \\
0 \quad r \geq 2^{1 / 6}+\Delta_{i j} \\
0 \quad r \leq \Delta_{i j} \\
U_{y u k}(r)=-\frac{\varepsilon_{i j, y}}{r-\Delta_{i j}} \exp \left(\kappa_{i j}\left(\Delta_{i j}+1-r\right)\right) \quad \Delta_{i j}<r<r_{c u t}+\Delta_{i j} \\
0 \quad r \geq r_{c u t}+\Delta_{i j}
\end{array}
\end{gathered}
$$

The shift, $\Delta_{\mathrm{ij}}$, between particles $i$ and $j$ is defined as $\Delta_{\mathrm{ij}}=\left(\sigma_{\mathrm{i}}+\sigma_{\mathrm{j}}\right) / 2-1$ such that the WCA potential provides a repulsive wall with the same slope regardless of the individual particle diameters. We chose $\kappa_{\mathrm{ec}}=\kappa_{\mathrm{ee}}=3.00 \sigma^{-1}$, and $\varepsilon_{\mathrm{ce}}=2.74$ and $\varepsilon_{\mathrm{ee}}=1.47$ to mimic a shortranged attraction with a minimum in the potential well of -2.00 and $\varepsilon_{\min }=-1.00$ for the rod end - colloid and rod end - rod end interaction, respectively. The magnitude of $\varepsilon_{\min }$ serves as the energy scale for our simulations, which we simply refer to as $\varepsilon$. The cutoff for the Yukawa tail 
was $r_{\text {cut,ce }}=3.00 \sigma, r_{\text {cut,ee }}=2.50 \sigma$. DNA-coated nanoparticles are coarse-grained to a single sphere with a diameter, $\sigma_{\mathrm{c}}$, whose interaction with all other spheres, besides end monomers, is exclusively given by the WCA potential. All other interactions are purely repulsive between all species, also given by the WCA potential above. The only exceptions to this are interactions involving shell particles. The seven spheres, equally spaced around each of the ends on each rod (cf. Figure 3), provide the rods with effectively directional interactions. These spheres have WCA repulsion with other end beads and the DNA-nanoparticles, but otherwise have no interaction with any other species. Thus, these shell particles reduce the propensity of rods to form pairs aligned parallel to each other, and inhibit the rod from laying perpendicular to the surface of the DNA nanoparticle when interacting with the surface. Both of these attributes are physically motivated by the nature of the ssDNA interactions which, because of the high energy cost of bending the hybridized ssDNA, are expected to have the same effects. Indeed, without these "directional" interactions, we did not observe the same qualitative phenomena we report on here.

We used the Highly-Optimized Object-Oriented Molecular Dynamics (HOOMD-blue) engine to perform our molecular dynamics simulations on graphics processing units (GPUs). We performed canonical (NVT) simulations where the temperature, volume and number of particles are constant during the simulation. Temperature was controlled with a Nose-Hoover thermostat with damping constant of $\tau=1.00$, and timestep between 0.001 and $0.005\left(m \sigma^{2} \varepsilon^{-1}\right)^{1 / 2}$ depending on system size, where $m$ is the mass of a particle. This was set to $m=1.00$ for all species in the system. We simulated 125 DNA-coated spherical nanoparticles and 1,250 rods (10:1 stoichiometry) in cubic simulation boxes whose length, $L$, depended on the colloid diameter. For $\sigma_{\mathrm{c}}=4.00 \sigma, 6.95 \sigma, 10.00 \sigma(16.00 \mathrm{~nm}, 27.80 \mathrm{~nm}, 40.00 \mathrm{~nm}$, see attached table $)$ we employed $L=63.30 \sigma, 110.00 \sigma, 158.00 \sigma$. This corresponds to a sphere volume fraction, $\phi_{\mathrm{s}}=\pi N_{\mathrm{c}} \sigma_{\mathrm{c}}{ }^{3} /\left(6 L^{3}\right)$, and rod volume fraction, $\phi_{\mathrm{r}}=\pi N_{\mathrm{r}} \sigma^{2} l_{\mathrm{rod}} /\left(4 L^{3}\right)$, were always less than 0.025 . Systems were initialized in a large box, then compressed to their final size at a reduced temperature $T^{*}=k_{\mathrm{B}} T / \varepsilon=1.00$ at which temperature they generally remained unstructured and isotropic. After an equilibration period of $5 \times 10^{6}$ timesteps, they were quenched to the desired $T^{*}$ and annealed for at least $2 \times 10^{8}$ timesteps, after which no qualitative changes were observed. We 
report results for $T^{*}=0.12$, however, we considered a range of $0.10 \leqslant T^{*} \leqslant 0.20$ over which we observed crystallization in this time frame and we found these results to be representative.

\section{Theory}

We posit that the primary difference a rod inside a close-packed crystal will sense between the polymorphs is the interference that exists between a rod attached to the surface of a sphere in one plane and a rod attached to a sphere the next-nearest stacking plane. In the FCC crystal, since

these planes are staggered (ABC pattern), rods on spheres in these planes only interact obliquely since they exist in different "channels" as depicted in Figure 4 (red and blue), whereas in the HCP, because the planes are eclipsed (ABAB pattern), a pair of rods often shares the same (blue) interplanar channel. If we reduce this to a one-dimensional model, considering a lattice onto which two rods are placed, we can contrast the two crystals.

We consider there to be $D$ lattice sites in the channel that exists between the surfaces of spheres in every other plane, where each site has length $\sigma$, such that a single bead from a rod fits on a site. From geometry we have (when rounded down to the nearest integer):

$$
D=\left\lfloor 2 \sqrt{\frac{2}{3}}\left(\sigma_{c}+l_{r o d}\right)-\sigma_{c}\right\rfloor
$$

This lattice is non-periodic and species cannot overlap the boundaries. In the FCC crystal, there are $D-l_{\text {rod }}+1$ unique positions for a single rod in a channel. If we consider a pair of rods, each in a different channel, then the total number of unique arrangements is:

$$
W_{f c c}=\left(D-l_{\text {rod }}+1\right)^{2}
$$

In contrast, in the HCP crystal, pairs of rods share the same channel. If rods cannot overlap then the total number of unique configurations can be counted by placing the first rod at every possible location starting from one end of the lattice, going to half way between the planes, and 
counting the total number of locations to place the second rod at every position for the first. By only placing the first rod systematically in the first half of the lattice, we avoid double counting configurations that would not be unique if we account for the rods being indistinguishable in the HCP crystal. In the FCC we assume the distinguishability of the 2 channels confers distinguishability of the rods each contains since they are assumed to remain fixed in each one's original channel. However, relaxation of this assumption contributes a $1 / 2$ ! term to the expression for $W_{\text {fcc }}$ which ultimately makes no qualitative changes to our results. If the number of lattice sites between the end of the first rod and the surface of the sphere is $x$, then it can be shown that the total number of configurations is:

$$
W_{h c p}=\sum_{x=0}^{\min \left(D-2 l_{\text {rod }}, l_{\text {rod }}-1\right)}\left(D-2 l_{\text {rod }}-x+1\right)+\sum_{x=l_{\text {rod }}}^{\left(D-l_{\text {rod }}\right) / 2} D-3 l_{\text {rod }}+2
$$

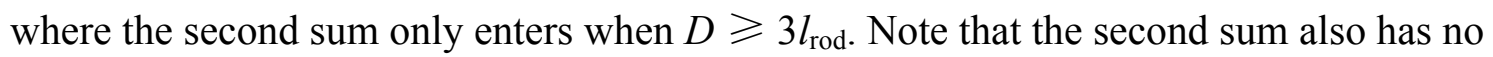
dependence on $x$, which is a consequence of how we formulate the counting of each state. It results because the second sum enters when the second rod may now come between the first rod and the edge of the lattice (sphere's surface) which is only possible when $D \geqslant 3 l_{\text {rod. }}$. The first sum depends on $x$ because it effectively reduces the number of locations available for the second rod's center of mass when $x$ is sufficiently small to prevent the second rod from coming between the first rod and the sphere's surface. However, when $x$ is large enough, it no longer affects the number of positions the second rod can exist in. Consequently, these sums may be compactly expressed as the sum of two products where the second only enters when $D \geqslant 3 l_{\text {rod. }}$

$$
W_{h c p}=\frac{1}{2}(1+\psi)\left(2\left(D-2 l_{r o d}+1\right)-\psi\right)+\left(\frac{D-l_{\text {rod }}}{2}-l_{\text {rod }}+1\right)\left(D-3 l_{\text {rod }}+2\right)
$$

where $\psi=\min \left(D-2 l_{\text {rod }}, l_{\text {rod }}-1\right)$. Note that we implicitly require $\psi>0$ which is simply a formal requirement that $l_{\text {rod }}>1$ and that $D \geqslant 2 l_{\text {rod. }}$. When the second condition is violated the lattice is too small to accommodate two rods in the same channel simultaneously, and the model's behavior on the HCP lattice is undefined. 
For each configuration, we can compute the total interaction energy using the potentials we employed in the MD simulations between different rod ends, and between the rod ends and the spheres. From this we obtain a Boltzmann factor which defines the un-normalized probability of that state. By summing over all $W$ states we can normalize this and obtain the energy and entropy according to:

$$
\begin{aligned}
& \hat{p}_{i}=\frac{\exp \left(-U_{i} / T\right)}{\sum_{i=0}^{W} \exp \left(-U_{i} / T\right)} \\
& \left\langle U_{\text {tot }}\right\rangle=\sum_{i=0}^{W} \hat{p}_{i} U_{i} \\
& S=-\sum_{i=0}^{W} \hat{p}_{i} \ln \hat{p}_{i}
\end{aligned}
$$

Therefore, the free energy, $F$, which is minimized at thermodynamic equilibrium in the canonical (NVT) ensemble, is easily obtained where $F=U-T S$.

\section{Design, Synthesis and Purification of DNA}

The sequences of DNA strands were designed using the program SEQUIN ${ }^{71}$. All DNA strands used in this study were purchased from Integrated DNA Technologies, Inc. (www.idtdna.com), and purified using $10 \%$ denaturing polyacrylamide gel electrophoresis. The concentrations of purified DNA strands were estimated by OD260.

\section{Denaturing Polyacrylamide Gel Electrophoresis}

The gels containing 10\% acrylamide (19:1, acrylamide/bisacrylamide) and 8.3 $\mathrm{M}$ urea were run at $55{ }^{\circ} \mathrm{C}$ and $600 \mathrm{~V}$ (constant voltage) on a Hoefer SE 600 electrophoresis unit. The running buffer consists of $100 \mathrm{mM}$ Tris- $\mathrm{HCl}$ (pH 8.3), $89 \mathrm{mM}$ Boric acid, and $2 \mathrm{mM}$ EDTA (TBE). The 
sample buffer is composed of $10 \mathrm{mM} \mathrm{NaOH}, 1 \mathrm{mM}$ EDTA, and trace amount of Bromophenol Blue and Xylene Cyanol FF tracking dye.

\section{Nondenaturing Polyacrylamide Gel Electrophoresis}

The gels containing 6\% acrylamide (19:1, acrylamide/bisacrylamide) were run on a Hoefer SE600 gel electrophoresis unit at a constant voltage of $200 \mathrm{~V}$ at room temperature. The running buffer consists of $40 \mathrm{mM}$ Tris- $\mathrm{HCl}$ (pH 8.0), $20 \mathrm{mM}$ acetate acid, $2 \mathrm{mM}$ EDTA, and $12.5 \mathrm{mM}$ magnesium acetate $\left(1 \times \mathrm{TAE} / \mathrm{Mg}^{2+}\right)$. Tracking dye containing $1 \times \mathrm{TAE} / \mathrm{Mg}^{2+}, 50 \%$ glycerol, and $0.02 \%$ each of Bromophenol Blue and Xylene Cyanol FF was added to the sample buffer. The gels were then stained with ethidium bromide and visualized with a Bio-Rad Universal Hood II gel imaging station.

\section{Preparation of DNA-gold NP conjugates}

The thiol-modified oligonucleotides were activated using tris [2-carboxyethyl] phosphine (TCEP) (100 : 1 molar ratio of TCEP: DNA) in water for $2 \mathrm{~h}$, followed by purification using G-25 size exclusion columns (GE Healthcare) to remove small molecules. Then the thiolated oligonucleotides were added into the aqueous nanoparticle solution at a DNA/NP ratio of $100: 1$ for $6 \mathrm{~nm} \mathrm{NPs}$, and 300 : 1 for $8.7 \mathrm{~nm}$ NPs. After 2h, the solution was brought to $\mathrm{pH} 7.4 \mathrm{of} 1 \times$ phosphate buffered saline (PBS: $100 \mathrm{mM} \mathrm{NaCl}, 10 \mathrm{mM} \mathrm{PB}$ ), and allowed to age for $2 \mathrm{~h}$. In the subsequent stepwise salt aging process, A salting solution containing $2 \mathrm{M} \mathrm{NaCl}$ was gradually added to the colloid solution to reach a final $\mathrm{NaCl}$ concentration of $0.3 \mathrm{M}$ (five steps with a $1 \mathrm{~h}$ waiting period between each addition), and the solution was allowed to age for another $12 \mathrm{~h}$. To remove unbound DNA, the solution was centrifuged, and the supernatant was pipetted off. The DNA-gold nanoparticle conjugates were washed with $0.1 \mathrm{M}$ PBS buffer $(0.1 \mathrm{M} \mathrm{NaCl}, 10 \mathrm{mM}$ phosphate buffer) and recentrifuged for 4 times. Finally, the functionalized nanoparticles were resuspended in freshly prepared 0.1 M PBS buffer, and the concentration was estimated from the optical absorbance at $\sim 520 \mathrm{~nm}$.

\section{Self-assembly of DNA 6HB rods}


The rods were formed by mixing a stoichiometric quantity of each DNA strand, as estimated by $\mathrm{OD}_{260}$, in a solution containing $40 \mathrm{mM}$ Tris- $\mathrm{HCl}, \mathrm{pH} 8.0,20 \mathrm{mM}$ acetic acid, $2.5 \mathrm{mM}$ EDTA, and $12.5 \mathrm{mM}$ magnesium acetate $\left(\mathrm{TAE} / \mathrm{Mg}^{2+}\right)$. The mixture was slowly cooled from $90{ }^{\circ} \mathrm{C}$ to room temperature over the course of $48 \mathrm{~h}$ in a $\sim 2 \mathrm{~L}$ water bath insulated in a Styrofoam box. The final concentration of each strand was $0.5 \mu \mathrm{M}$.

\section{Self-assembly of nanoparticle superlattices}

Particle assembly was carried out by combining a specified ratio of the 6HB rods and the DNAcapped gold NPs in a solution containing $40 \mathrm{mM}$ Tris-HCl, $\mathrm{pH} 8.0,20 \mathrm{mM}$ acetic acid, $2.5 \mathrm{mM}$ EDTA, and $12.5 \mathrm{mM}$ magnesium acetate $\left(\mathrm{TAE} / \mathrm{Mg}^{2+}\right)$. The mixture was slowly cooled over $\sim 53 \mathrm{~h}$ from $51^{\circ} \mathrm{C}$ to $25^{\circ} \mathrm{C}$ in an incubator (Echotherm TM IN35)

\section{Small Angle X-ray scattering (SAXS)}

SAXS was performed at National Synchrotron Light Source (NSLS) X-9 beamline. The scattering data were collected with a CCD area detector, and then integrated into a $1 \mathrm{D}$ scattering curve $\mathrm{I}(q)$ as a function of the scattering vector $\mathrm{q}$, where $\mathrm{q}=(4 \pi / \lambda) \sin (\theta / 2)$, with $\lambda$ and $\theta$ being the wavelength of incident X-rays and the scattering angle, respectively. The structure factor $\mathrm{S}(q)$ was obtained by dividing $\mathrm{I}(q)$ by the particle form factor $\mathrm{F}(q)$ determined from a suspension of unaggregated free particles ${ }^{7}$.

\section{Supporting Information}

The Supporting Information is available free of charge on the ACS Publications website at DOI: XXXXXX

- Calculation of experimental nanoparticle sizes and interparticle spacing

- DNA sequences used to create the origami rods and capping sequences on the spheres

- Salt stress size-dependence of rod aggregates

\section{$\underline{\text { Acknowledgement }}$}


Research carried out at the Center for Functional Nanomaterials, Brookhaven National Laboratory, was supported by the US Department of Energy, Office of Basic Energy Sciences (contract no. DE-SC0012704). This work was supported by the US Department of Energy, Office of Basic Energy Sciences, grant DE-SC0008772 (Columbia), and by the collaborative research grant CBET-1402166 (Princeton) from the U.S. National Science Foundation. In addition, NAM gratefully acknowledges support from a National Research Council postdoctoral research associateship at the National Institute of Standards and Technology. 
1. Sun, S.; Murray, C. B.; Weller, D.; Folks, L.; Moser, A., Monodisperse Fept Nanoparticles and Ferromagnetic Fept Nanocrystal Superlattices. Science 2000, 287 (5460), 1989-92.

2. Rockstuhl, C.; Lederer, F.; Etrich, C.; Pertsch, T.; Scharf, T., Design of an Artificial ThreeDimensional Composite Metamaterial with Magnetic Resonances in the Visible Range of the Electromagnetic Spectrum. Phys. Rev. Lett. 2007, 99 (1), 017401.

3. Mann, S., Self-Assembly and Transformation of Hybrid Nano-Objects and Nanostructures under Equilibrium and Non-Equilibrium Conditions. Nat Mater 2009, 8 (10), 781-92.

4. Glotzer, S. C.; Solomon, M. J., Anisotropy of Building Blocks and Their Assembly into Complex Structures. Nat Mater 2007, 6 (8), 557-62.

5. Tao, A.; Sinsermsuksakul, P.; Yang, P., Tunable Plasmonic Lattices of Silver Nanocrystals. Nat Nanotechnol 2007, 2 (7), 435-40.

6. Stebe, K. J.; Lewandowski, E.; Ghosh, M., Materials Science. Oriented Assembly of Metamaterials. Science 2009, 325 (5937), 159-60.

7. Nykypanchuk, D.; Maye, M. M.; van der Lelie, D.; Gang, O., DNA-Guided Crystallization of Colloidal Nanoparticles. Nature 2008, 451 (7178), 549-552.

8. Maye, M. M.; Nykypanchuk, D.; Cuisinier, M.; van der Lelie, D.; Gang, O., Stepwise Surface Encoding for High-Throughput Assembly of Nanoclusters. Nat Mater 2009, 8 (5), 388-91.

9. Xiong, H.; van der Lelie, D.; Gang, O., Phase Behavior of Nanoparticles Assembled by DNA Linkers. Phys. Rev. Lett. 2009, 102 (1), 015504.

10. Park, S. Y.; Lytton-Jean, A. K.; Lee, B.; Weigand, S.; Schatz, G. C.; Mirkin, C. A., DNAProgrammable Nanoparticle Crystallization. Nature 2008, 451 (7178), 553-6.

11. Macfarlane, R. J.; Lee, B.; Jones, M. R.; Harris, N.; Schatz, G. C.; Mirkin, C. A., Nanoparticle Superlattice Engineering with DNA. Science 2011, 334 (6053), 204-8.

12. Kostiainen, M. A.; Hiekkataipale, P.; Laiho, A.; Lemieux, V.; Seitsonen, J.; Ruokolainen, J.; Ceci, P., Electrostatic Assembly of Binary Nanoparticle Superlattices Using Protein Cages. Nat Nanotechnol 2013, $8(1), 52-6$.

13. Zhao, Y.; Thorkelsson, K.; Mastroianni, A. J.; Schilling, T.; Luther, J. M.; Rancatore, B. J.; Matsunaga, K.; Jinnai, H.; Wu, Y.; Poulsen, D.; Frechet, J. M.; Alivisatos, A. P.; Xu, T., Small-MoleculeDirected Nanoparticle Assembly Towards Stimuli-Responsive Nanocomposites. Nat Mater 2009, 8 (12), 979-85.

14. Tang, Z.; Zhang, Z.; Wang, Y.; Glotzer, S. C.; Kotov, N. A., Self-Assembly of Cdte Nanocrystals into Free-Floating Sheets. Science 2006, 314 (5797), 274-8.

15. Leunissen, M. E.; Christova, C. G.; Hynninen, A. P.; Royall, C. P.; Campbell, A. I.; Imhof, A.; Dijkstra, M.; van Roij, R.; van Blaaderen, A., Ionic Colloidal Crystals of Oppositely Charged Particles. Nature 2005, 437 (7056), 235-240.

16. Shevchenko, E. V.; Talapin, D. V.; Kotov, N. A.; O'Brien, S.; Murray, C. B., Structural Diversity in Binary Nanoparticle Superlattices. Nature 2006, 439 (7072), 55-59.

17. Damasceno, P. F.; Engel, M.; Glotzer, S. C., Predictive Self-Assembly of Polyhedra into Complex Structures. Science 2012, 337 (6093), 453-457.

18. Dussi, S.; Dijkstra, M., Entropy-Driven Formation of Chiral Nematic Phases by Computer Simulations. Nature Communications 2016, 7.

19. Reinhardt, A.; Frenkel, D., DNA Brick Self-Assembly with an Off-Lattice Potential. Soft Matter 2016, 12 (29), 6253-6260.

20. Zanjani, M. B.; Jenkins, I. C.; Crocker, J. C.; Sinno, T., Colloidal Cluster Assembly into Ordered Superstructures Via Engineered Directional Binding. Acs Nano 2016, 10 (12), 11280-11289.

21. Zhang, J.; Luijten, E.; Granick, S., Toward Design Rules of Directional Janus Colloidal Assembly. In Annual Review of Physical Chemistry, Vol 66, Johnson, M. A.; Martinez, T. J., Eds. 2015; Vol. 66, pp 581600. 
22. Seeman, N. C., DNA in a Material World. Nature 2003, 421 (6921), 427-31.

23. Winfree, E.; Liu, F.; Wenzler, L. A.; Seeman, N. C., Design and Self-Assembly of Two-Dimensional DNA Crystals. Nature 1998, 394 (6693), 539-44.

24. Yan, H.; Park, S. H.; Finkelstein, G.; Reif, J. H.; LaBean, T. H., DNA-Templated Self-Assembly of Protein Arrays and Highly Conductive Nanowires. Science 2003, 301 (5641), 1882-1884.

25. Ding, B.; Sha, R.; Seeman, N. C., Pseudohexagonal 2d DNA Crystals from Double Crossover Cohesion. J. Am. Chem. Soc. 2004, 126 (33), 10230-1.

26. Ke, Y.; Liu, Y.; Zhang, J.; Yan, H., A Study of DNA Tube Formation Mechanisms Using 4-, 8-, and 12-Helix DNA Nanostructures. J. Am. Chem. Soc. 2006, 128 (13), 4414-21.

27. Liu, D.; Wang, M.; Deng, Z.; Walulu, R.; Mao, C., Tensegrity: Construction of Rigid DNA Triangles with Flexible Four-Arm DNA Junctions. J. Am. Chem. Soc. 2004, 126 (8), 2324-5.

28. He, Y.; Ye, T.; Su, M.; Zhang, C.; Ribbe, A. E.; Jiang, W.; Mao, C., Hierarchical Self-Assembly of DNA into Symmetric Supramolecular Polyhedra. Nature 2008, 452 (7184), 198-201.

29. Liu, W.; Zhong, H.; Wang, R.; Seeman, N. C., Crystalline Two-Dimensional DNA-Origami Arrays. Angew. Chem. Int. Ed. Engl. 2011, 50 (1), 264-7.

30. Zheng, J.; Birktoft, J. J.; Chen, Y.; Wang, T.; Sha, R.; Constantinou, P. E.; Ginell, S. L.; Mao, C.; Seeman, N. C., From Molecular to Macroscopic Via the Rational Design of a Self-Assembled 3d DNA Crystal. Nature 2009, 461 (7260), 74-7.

31. Park, S. H.; Yin, P.; Liu, Y.; Reif, J. H.; LaBean, T. H.; Yan, H., Programmable DNA Self-Assemblies for Nanoscale Organization of Ligands and Proteins. Nano Lett. 2005, 5 (4), 729-33.

32. Liu, Y.; Lin, C.; Li, H.; Yan, H., Aptamer-Directed Self-Assembly of Protein Arrays on a DNA Nanostructure. Angew. Chem. Int. Ed. Engl. 2005, 44 (28), 4333-8.

33. Xiao, S. L., F.; Rosen, A. E.; Hainfeld, J. F.; Seeman, N. C; Musier-Forsyth, K.; Kiehl, R. A., Selfassembly of Metallic Nanoparticle Arrays by DNA Scaffolding. J. Nanopart. Res. 2002, 4, 313-317.

34. Le, J. D. P., Y.; Seeman, N. C; Musier-Forsyth, K.; Taton, T. A.; Kiehl, R. A., DNA-Templated SelfAssembly of Metallic Nanocomponent Arrays on a Surface. Nano Lett. 2004, 4, 2343-2347.

35. Zhang, J.; Liu, Y.; Ke, Y.; Yan, H., Periodic Square-Like Gold Nanoparticle Arrays Templated by Self-Assembled 2d DNA Nanogrids on a Surface. Nano Lett. 2006, 6 (2), 248-51.

36. Sharma, J.; Chhabra, R.; Cheng, A.; Brownell, J.; Liu, Y.; Yan, H., Control of Self-Assembly of DNA Tubules through Integration of Gold Nanoparticles. Science 2009, 323 (5910), 112-6.

37. Cadotte, A. T.; Dshemuchadse, J.; Damasceno, P. F.; Newman, R. S.; Glotzer, S. C., Self-Assembly of a Space-Tessellating Structure in the Binary System of Hard Tetrahedra and Octahedra. Soft Matter 2016, 12 (34), 7073-7078.

38. Adams, M.; Dogic, Z.; Keller, S. L.; Fraden, S., Entropically Driven Microphase Transitions in Mixtures of Colloidal Rods and Spheres. Nature 1998, 393 (6683), 349-352.

39. Ahmad, I.; Zandvliet, H. J. W.; Kooij, E. S., Shape-Induced Separation of Nanospheres and Aligned Nanorods. Langmuir 2014, 30 (27), 7953-7961.

40. Dogic, Z.; Frenkel, D.; Fraden, S., Enhanced Stability of Layered Phases in Parallel Hard Spherocylinders Due to Addition of Hard Spheres. Physical Review E 2000, 62 (3), 3925-3933.

41. Radin, C.; Sadun, L., Structure of the Hard Sphere Solid. Phys. Rev. Lett. 2005, 94 (1).

42. Woodcock, L. V., Entropy Difference between the Face-Centred Cubic and Hexagonal ClosePacked Crystal Structures. Nature 1997, 385 (6612), 141-143.

43. Tkachenko, A. V., Theory of Programmable Hierarchic Self-Assembly. Phys. Rev. Lett. 2011, 106 (25).

44. Leunissen, M. E.; Frenkel, D., Numerical Study of DNA-Functionalized Microparticles and Nanoparticles: Explicit Pair Potentials and Their Implications for Phase Behavior. J. Chem. Phys. 2011, 134 (8). 
45. Knorowski, C.; Travesset, A., Materials Design by DNA Programmed Self-Assembly. Current Opinion in Solid State \& Materials Science 2011, 15 (6), 262-270.

46. Park, S. Y.; Lytton-Jean, A. K. R.; Lee, B.; Weigand, S.; Schatz, G. C.; Mirkin, C. A., DNAProgrammable Nanoparticle Crystallization. Nature 2008, 451 (7178), 553-556.

47. Angioletti-Uberti, S.; Mognetti, B. M.; Frenkel, D., Theory and Simulation of DNA-Coated Colloids: A Guide for Rational Design. PCCP 2016, 18 (9), 6373-6393.

48. Li, T.; Sknepnek, R.; de la Cruz, M. O., Thermally Active Hybridization Drives the Crystallization of DNA-Functionalized Nanoparticles. J. Am. Chem. Soc. 2013, 135 (23), 8535-8541.

49. Li, T.; Sknepnek, R.; Macfarlane, R. J.; Mirkin, C. A.; de la Cruz, M. O., Modeling the Crystallization of Spherical Nucleic Acid Nanoparticle Conjugates with Molecular Dynamics Simulations. Nano Lett. 2012, 12 (5), 2509-2514.

50. Srinivasan, B.; Vo, T.; Zhang, Y. G.; Gang, O.; Kumar, S.; Venkatasubramanian, V., Designing DNAGrafted Particles That Self-Assemble into Desired Crystalline Structures Using the Genetic Algorithm. Proceedings of the National Academy of Sciences of the United States of America 2013, 110 (46), 1843118435.

51. Vo, T.; Venkatasubramanian, V.; Kumar, S.; Srinivasan, B.; Pal, S.; Zhang, Y. G.; Gang, O., Stoichiometric Control of DNA-Grafted Colloid Self-Assembly. Proceedings of the National Academy of Sciences of the United States of America 2015, 112 (16), 4982-4987.

52. Vial, S.; Nykypanchuk, D.; Yager, K. G.; Tkachenko, A. V.; Gang, O., Linear Mesostructures in DNA-Nanorod Self-Assembly. Acs Nano 2013, 7 (6), 5437-5445.

53. Lu, F.; Yager, K. G.; Zhang, Y.; Xin, H.; Gang, O., Superlattices Assembled through Shape-Induced Directional Binding. Nat Commun 2015, 6, 6912.

54. Douglas, S. M.; Dietz, H.; Liedl, T.; Hogberg, B.; Graf, F.; Shih, W. M., Self-Assembly of DNA into Nanoscale Three-Dimensional Shapes (Vol 459, Pg 414, 2009). Nature 2009, 459 (7250), 1154-1154.

55. Liu, W.; Tagawa, M.; Xin, H. L.; Wang, T.; Emamy, H.; Li, H.; Yager, K. G.; Starr, F. W.; Tkachenko, A. V.; Gang1, O., Diamond Family of Nanoparticle Superlattices. Science 2016, 351 (6273), 582-586.

56. Tian, Y.; Zhang, Y.; Wang, T.; Xin, H. L.; Li, H.; Gang, O., Lattice Engineering through Nanoparticle-DNA Frameworks. Nature Materials 2016.

57. Wang, T.; Schiffels, D.; Cuesta, S. M.; Fygenson, D. K.; Seeman, N. C., Design and Characterization of $1 \mathrm{~d}$ Nanotubes and 2d Periodic Arrays Self-Assembled from DNA Multi-Helix Bundles. J. Am. Chem. Soc. 2012, 134 (3), 1606-16.

58. Mathieu, F.; Liao, S.; Kopatsch, J.; Wang, T.; Mao, C.; Seeman, N. C., Six-Helix Bundles Designed from DNA. Nano Lett. 2005, 5 (4), 661-5.

59. Kuzuya, A.; Wang, R.; Sha, R.; Seeman, N. C., Six-Helix and Eight-Helix DNA Nanotubes Assembled from Half-Tubes. Nano Lett. 2007, 7 (6), 1757-63.

60. Wang, R.; Liu, W.; Seeman, N. C., Prototyping Nanorod Control: A DNA Double Helix Sheathed within a DNA Six-Helix Bundle. Chem Biol 2009, 16 (8), 862-7.

61. Bellot, G.; McClintock, M. A.; Chou, J. J.; Shih, W. M., DNA Nanotubes for Nmr Structure Determination of Membrane Proteins. Nat Protoc 2013, 8 (4), 755-70.

62. Bloomfield, V. A., DNA Condensation by Multivalent Cations. Biopolymers 1997, 44 (3), 269-282.

63. Dai, L.; Mu, Y.; Nordenskioeld, L.; van der Maarel, J. R. C., Molecular Dynamics Simulation of Multivalent-Ion Mediated Attraction between DNA Molecules. Phys. Rev. Lett. 2008, 100 (11).

64. Delacruz, M. O.; Belloni, L.; Delsanti, M.; Dalbiez, J. P.; Spalla, O.; Drifford, M., Precipitation of Highly-Charged Polyelectrolyte Solutions in the Presence of Multivalent Salts. J. Chem. Phys. 1995, 103 (13), 5781-5791.

65. Qiu, X.; Parsegian, V. A.; Rau, D. C., Divalent Counterion-Induced Condensation of Triple-Strand DNA. Proceedings of the National Academy of Sciences of the United States of America 2010, 107 (50), 21482-21486. 
66. Solis, F. J.; de la Cruz, M. O., Attractive Interactions between Rodlike Polyelectrolytes: Polarization, Crystallization, and Packing. Physical Review E 1999, 60 (4), 4496-4499.

67. Chi, C.; Vargas-Lara, F.; Tkachenko, A. V.; Starr, F. W.; Gang, O., Internal Structure of Nanoparticle Dimers Linked by DNA. Acs Nano 2012, 6 (8), 6793-6802.

68. Auyeung, E.; Cutler, J. I.; Macfarlane, R. J.; Jones, M. R.; Wu, J.; Liu, G.; Zhang, K.; Osberg, K. D.; Mirkin, C. A., Synthetically Programmable Nanoparticle Superlattices Using a Hollow Three-Dimensional Spacer Approach. Nat Nanotechnol 2012, 7 (1), 24-8.

69. Gasser, U.; Weeks, E. R.; Schofield, A.; Pusey, P. N.; Weitz, D. A., Real-Space Imaging of Nucleation and Growth in Colloidal Crystallization. Science 2001, 292 (5515), 258-262.

70. Zhu, J. X.; Li, M.; Rogers, R.; Meyer, W.; Ottewill, R. H.; Russell, W. B.; Chaikin, P. M., Crystallization of Hard-Sphere Colloids in Microgravity. Nature 1997, 387 (6636), 883-885.

71. Seeman, N. C., De Novo Design of Sequences for Nucleic Acid Structural Engineering. J. Biomol. Struct. Dyn. 1990, 8 (3), 573-81.

\section{$\underline{\text { TOC Image }}$}

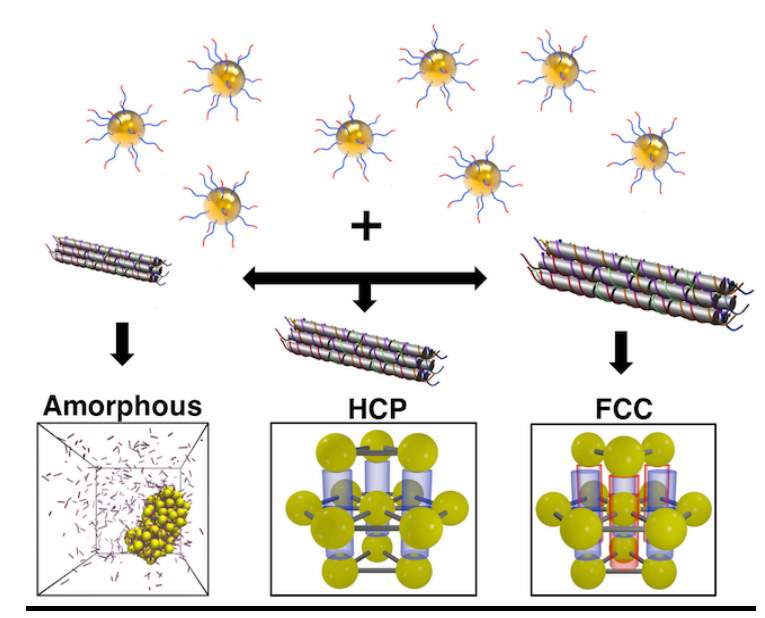

